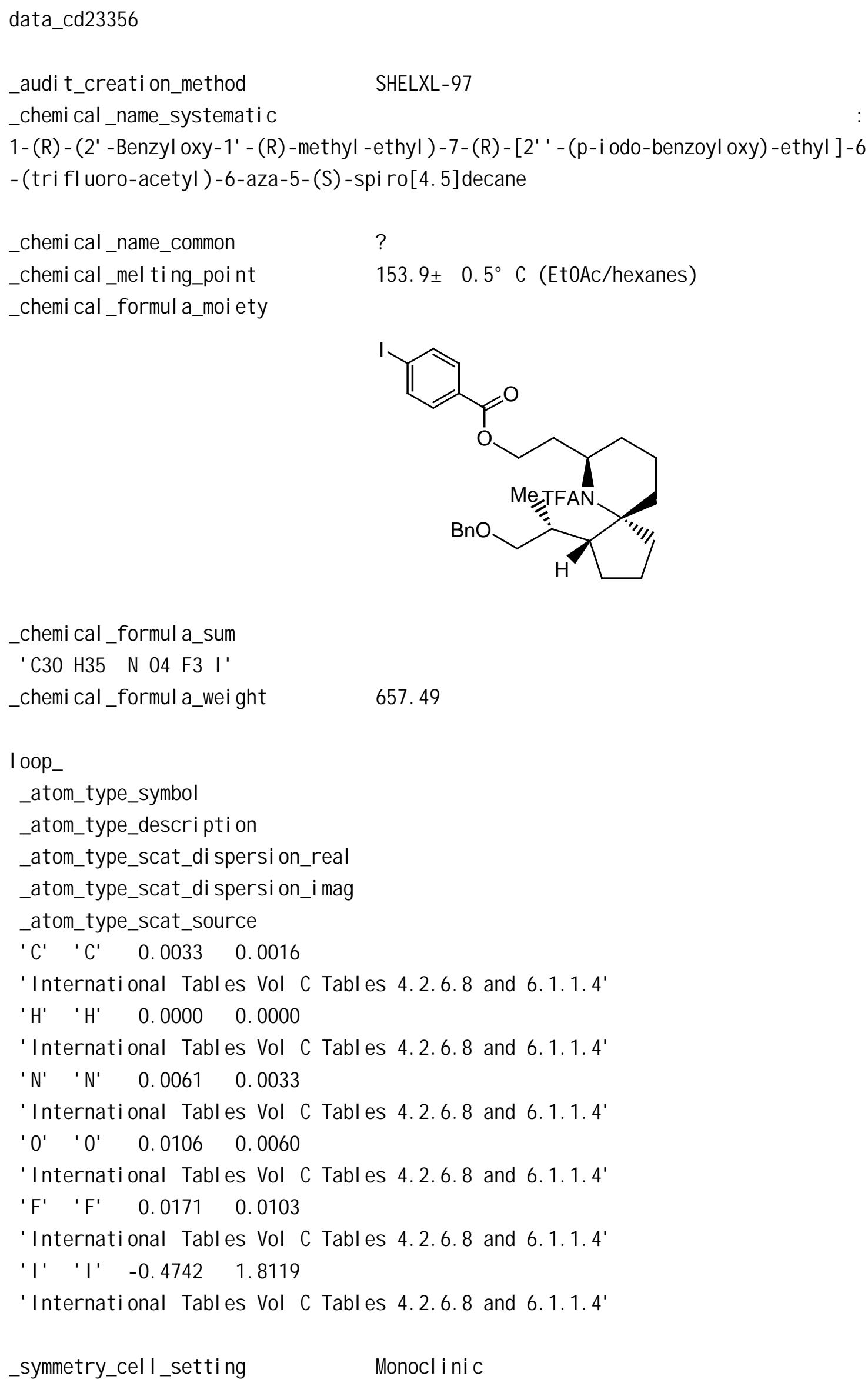

_chemical_f ormul a_sum

' $\mathrm{C} 30 \mathrm{H} 35$ N 04 F3 I'

chemical_f ormula_weight $\quad 657.49$

$100 p_{-}$

- at o m_t ype_symbol

_atom_type_description

_atom_type_scat_dispersion_real

_atom_type_scat_dispersion_imag

_atom_type_scat_source

$\begin{array}{llll}C^{\prime} & \text { ' } C & 0.0033 & 0.0016\end{array}$

'International Tables Vol C Tables 4.2.6.8 and 6.1.1.4'

' $\mathrm{H}^{\prime} \quad \mathrm{H}^{\prime} \quad 0.0000 \quad 0.0000$

'International Tables Vol C Tables 4.2.6.8 and 6.1.1.4'

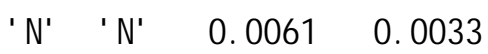

'International Tables Vol C Tables 4.2.6.8 and 6.1.1.4'

' 0 ' ' 0 ' $0.0106 \quad 0.0060$

'International Tables Vol C Tables 4.2.6.8 and 6.1.1.4'

$\begin{array}{llll}\text { ' } F^{\prime} & \text { 'F' } & 0.0171 & 0.0103\end{array}$

'International Tables Vol C Tables 4.2.6.8 and 6.1.1.4'

'I' 'I' $\quad .0 .4742 \quad 1.8119$

'International Tables Vol C Tables 4.2.6.8 and 6.1.1.4'

_symmetry_cell_setting Monoclinic 


\section{$100 p_{-}$}

_symmetry_space_group_name_H- M

_symmetry_equiv_pos_as_xyz

' $\mathrm{x}, \mathrm{y}, \mathrm{z}$ '

$' \cdot x, y+1 / 2,-z^{\prime}$

\begin{tabular}{|c|c|}
\hline _cell_length_a & $7.2203(7)$ \\
\hline _cell_length_b & $14.0238(13)$ \\
\hline _cell_length_c & $14.0714(13)$ \\
\hline _cell_angle_alpha & 90.00 \\
\hline _cell_angle_beta & $91.101(2)$ \\
\hline _cell_angle_gamma & 90.00 \\
\hline _cell_volume & $1424.6(2)$ \\
\hline _cell_formula_units_z & 2 \\
\hline _cell_measurement_temperature & $293(2)$ \\
\hline _cell_measurement_reflns_used & 3233 \\
\hline _cell_measurement_theta_mi n & 5.643 \\
\hline _cell_measurement_theta_max & 48.928 \\
\hline _exptl_crystal_description & $?$ \\
\hline _exptl_crystal_colour & colorless \\
\hline _exptl_crystal_size_max & 0.533 \\
\hline _exptl_crystal_size_mid & 0.418 \\
\hline _exptl_crystal_size_min & 0.217 \\
\hline _exptl_crystal_density_meas & $?$ \\
\hline _exptl_crystal_density_diffrn & 1.533 \\
\hline _exptl_crystal_density_method & 'not measured' \\
\hline _exptl_crystal_F_000 & 668 \\
\hline _exptl_absorpt_coefficient_mu & 1.179 \\
\hline _exptl_absorpt_correction_type & sadabs \\
\hline _exptl_absorpt_correction_ $T_{-}$min & 0.55470 \\
\hline _exptl_absorpt_correction_ $T_{-} \max$ & 1.00000 \\
\hline _exptl_absorpt_process_details & $?$ \\
\hline _exptl_special_details & \\
\hline ; & \\
\hline$?$ & \\
\hline 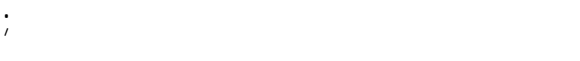 & \\
\hline _diffrn_ambient_temperature & $293(2)$ \\
\hline _diffrn_radiation_wavel ength & 0.71073 \\
\hline _diffrn_radiation_type & Mo K \a \\
\hline diffrn_radiation_source & 'fine-focus sealed tube' \\
\hline
\end{tabular}




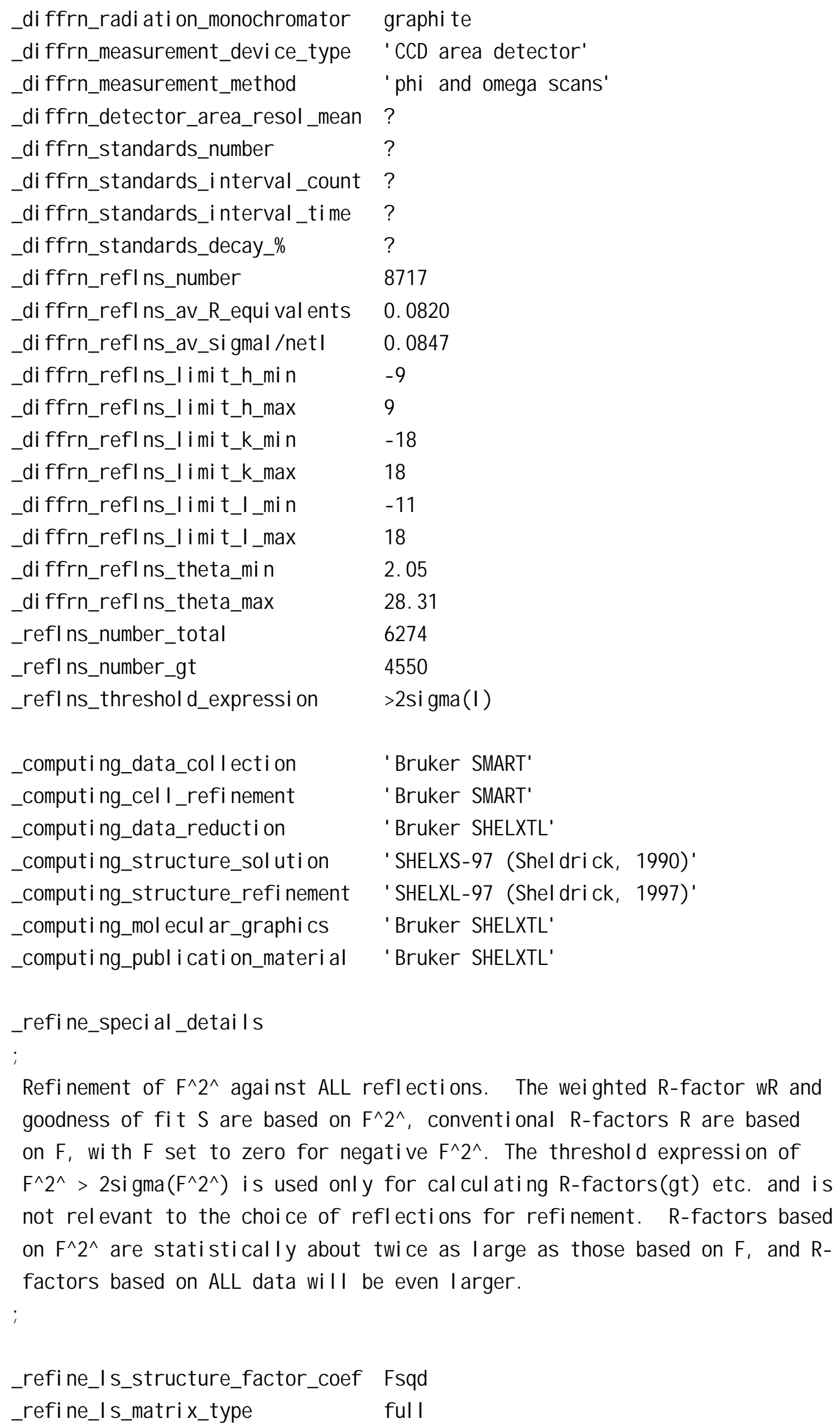




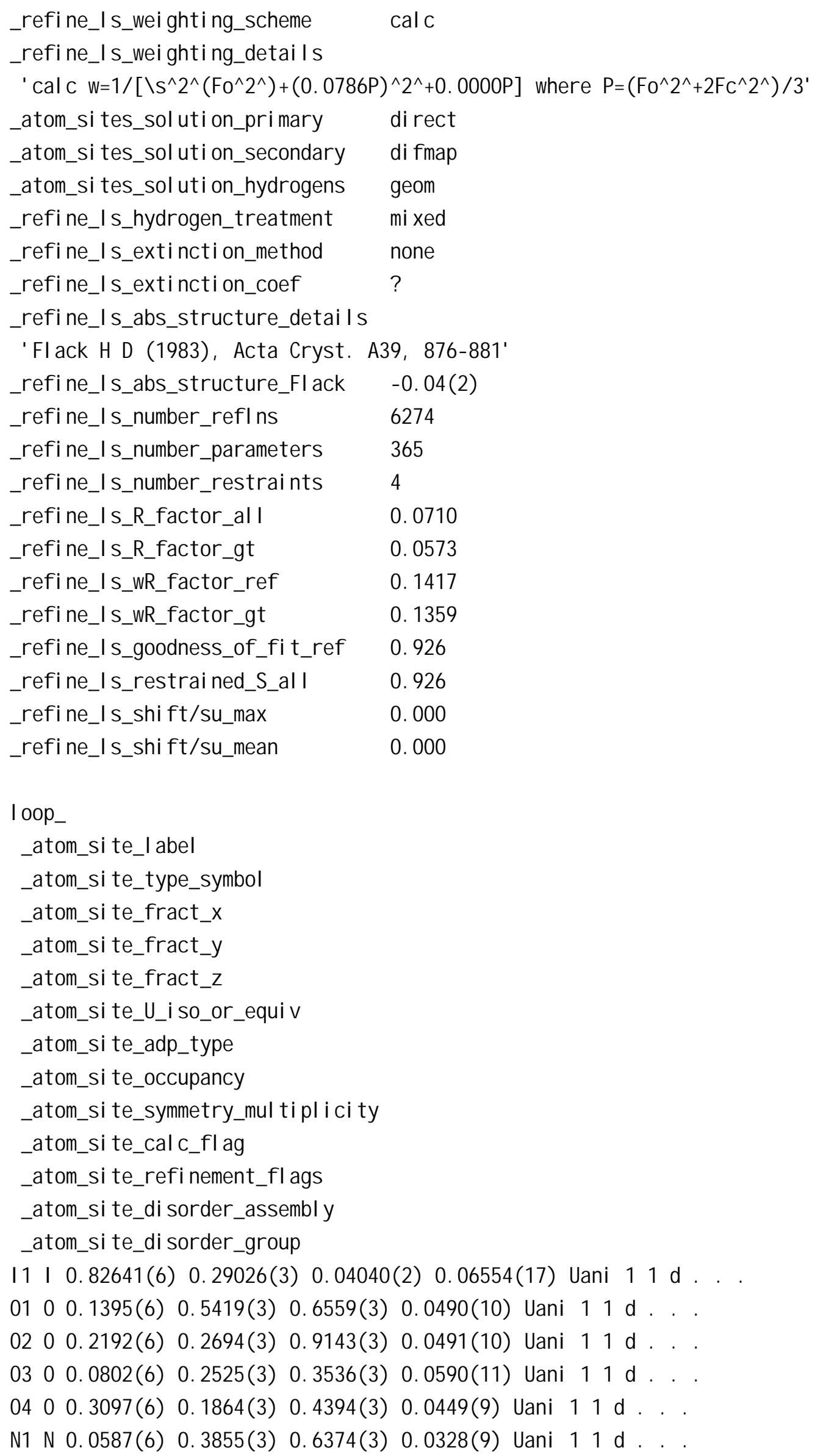


F1 F $0.3819(5) \quad 0.3912(3) \quad 0.5184(3) \quad 0.0615(10)$ Uani $11 \mathrm{~d} . \ldots$ F2 F $0.1775(6) 0.4745(3) 0.4454(2) 0.0708(12)$ Uani $11 \mathrm{~d} .$. F3 F $0.3862(7) 0.5415(3) 0.5306(3) 0.0745(12)$ Uani $11 \mathrm{~d} .$. C1 C $0.3007(7) \quad 0.3194(4) \quad 0.8378(4) \quad 0.0401(13)$ Uani $11 \mathrm{~d} . .$. $\begin{array}{llllllllllll}\text { H1A H } & 0.2717 & 0.2874 & 0.7782 & 0.048 & \text { Uiso } 1 & 1 & \text { calc } & R\end{array}$. H1B H $0.43430 .3206 \quad 0.84640 .048$ Uiso 11 calc $R$. . C2 C $0.2251(8) \quad 0.4213(4) \quad 0.8350(4) \quad 0.0381(11)$ Uani $11 \mathrm{~d} D$. C3 C $0.0174(8) 0.4247(4) 0.8176(4) 0.0381(11)$ Uani 11 d D.. C4 C $-0.0804(9) \quad 0.5197(4) \quad 0.8370(5) \quad 0.0510(15)$ Uani $11 \mathrm{~d} . .$. H4A H -0.08640 .53190 .90470 .061 Uiso 11 calc $R$. . H4 B H -0.01660 .57220 .80690 .061 Uiso $11 \mathrm{calc} R$. . C5 C - 0.2691(9) $0.5066(5) \quad 0.7946(5) \quad 0.0583(17)$ Uani $11 \mathrm{~d} .$. H5A H -0.33120 .56750 .78620 .070 Uiso $11 \mathrm{calc} R$. . H5B H $-0.3440 \quad 0.4660 \quad 0.83440 .070$ Uiso 11 calc $R$. . C6 C $-0.2347(9) \quad 0.4599(5) \quad 0.6999(5) \quad 0.0527(15)$ Uani $11 \mathrm{~d} .$. H6A H -0.20130 .50700 .65270 .063 Uiso 11 calc $R$. H6B H $-0.34380 .4257 \quad 0.67740 .063$ Uiso 11 calc $R$. C7 C $\cdot 0.0706(7) \quad 0.3896(4) \quad 0.7201(4) 0.0350(11)$ Uani $11 \mathrm{~d} . .$. C8 C $0.1468(6) 0.2880(5) 0.7357(3) \quad 0.0406(10)$ Uani $11 \mathrm{~d} .$. H8A H $\cdot 0.04490 .24700 .75550 .049$ Uiso 11 calc $R$. . H8 B H $-0.23380 .2900 \quad 0.78730 .049$ Uiso 11 calc $R$. C9 C - $0.2436(8) 0.2430(5) \quad 0.6490(5) \quad 0.0536(15)$ Uani $11 \mathrm{~d} .$. H9A H -0.22860 .17430 .65160 .064 Uiso $11 \mathrm{calc} R$. . H9B H -0.37510 .25690 .65060 .064 Uiso 11 calc $R$. C10 C - $0.1654(7) \quad 0.2802(7) \quad 0.5569(4) \quad 0.0493(15)$ Uani $11 \mathrm{~d} .$. H10A H -0.23480 .33610 .53670 .059 Uiso 11 calc $R$. H1OB H -0.17990 .23190 .50790 .059 Uiso 11 calc $R$. . C11 C $0.0395(7) \quad 0.3062(4) 0.5679(3)$ 0.0352(11) Uani $11 \mathrm{~d}$ D. . C12 C $0.1616(8) 0.2204(4) 0.5914(4) \quad 0.0395(12)$ Uani $11 \mathrm{~d} .$. H12A H 0.28350 .24280 .61100 .047 Uiso 11 calc $R$. . H12B H 0.10930 .18640 .64460 .047 Uiso 11 calc $R$. C13 C $0.1819(10) \quad 0.1519(4) \quad 0.5093(4) \quad 0.0486(14)$ Uani 11 d... H13A H 0.22510 .09090 .53340 .058 Uiso $11 \mathrm{calc} R$. . H13B H 0.06160 .14200 .47900 .058 Uiso 11 calc $R$. . C14 C $0.2396(8) \quad 0.2315(4) \quad 0.3627(4) \quad 0.0413(12)$ Uani $11 \mathrm{~d} . .$. C15 C $0.3822(7) \quad 0.2479(4) \quad 0.2908(4) \quad 0.0360(11)$ Uani $11 d . .$. C16 C $0.5636(8) \quad 0.2210(4) \quad 0.3037(4) \quad 0.0417(12)$ Uani $11 \mathrm{~d} . .$. $\begin{array}{llllllllll}H 16 & \text { H } & 0.6016 & 0.1943 & 0.3613 & 0.050 & \text { Uiso } 1 & 1 & \text { calc } & R\end{array}$. . C17 C $0.6896(8) \quad 0.2329(4) \quad 0.2333(4) \quad 0.0464(13)$ Uani $11 \mathrm{~d} . .$.

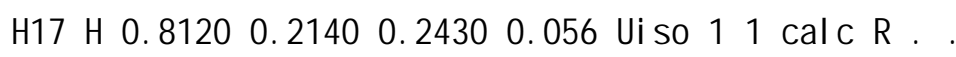
C18C $0.6340(7) \quad 0.2733(4) \quad 0.1472(4) \quad 0.0420(13)$ Uani $11 \mathrm{~d} . .$. C19C $0.4517(8) 0.3021(5) \quad 0.1339(4) \quad 0.0502(14)$ Uani $11 \mathrm{~d} . .$. H1 9 H 0.41430 .32970 .07660 .060 Uiso 11 calc $R$. . C20 C $0.3264(7) 0.2902(6) 0.2048(3) 0.0490(12)$ Uani $11 \mathrm{~d} .$. 
H2O H $0.20450 .31010 .1958 \quad 0.059$ Uiso 11 calc $R$.

C21 C $0.1477(7) 0.4671(4) 0.6140(3) 0.0355(11)$ Uani $11 \mathrm{~d} .$.

C22 C $0.2758(9) 0.4673(4) \quad 0.5265(4) \quad 0.0434(13)$ Uani $11 \mathrm{~d} .$.

C23 C $0.2809(10) \quad 0.4718(5) \quad 0.9266(5) \quad 0.0535(15)$ Uani $11 \mathrm{~d} .$.

H23A H 0.25490 .53870 .92070 .080 Uiso $11 \mathrm{calc} R$.

H23B H 0.41110 .46280 .93880 .080 Uiso 11 calc $R$. .

H23C H $0.21220 .4458 \quad 0.97830 .080$ Uiso 11 calc $R$. .

C24 C $0.3001(10) \quad 0.1785(5) \quad 0.9317(5) \quad 0.0577(16)$ Uani $11 \mathrm{~d} .$. H24A H $0.2550 \quad 0.15410 .99140 .069$ Uiso 11 calc $R$.

H24B H 0.43330 .18610 .93830 .069 Uiso 11 calc $R$. .

C25 C $0.2594(10) 0.1062(4) \quad 0.8546(5) 0.0577(17)$ Uani 11 d . .

C26 C $0.3926(15) \quad 0.0720(6) \quad 0.7984(6) \quad 0.084(2)$ Uani $11 \mathrm{~d} . .$.

H2 6 H 0.51340 .09460 .80440 .101 Uiso 11 calc $R$. .

C27C $0.347(2) 0.0007(8) 0.7292(8) 0.116(4)$ Uani $11 \mathrm{~d} .$.

H2 7 H $0.4395 \cdot 0.02550 .69230 .139$ Uiso $11 \mathrm{calc} R$. .

C28 C $0.173(3) \cdot 0.0285(8) \quad 0.7172(10) \quad 0.143(8)$ Uani $11 \mathrm{~d} .$.

H2 8 H $0.1433 \cdot 0.07290 .67030 .172$ Uiso 11 calc $\mathrm{R}$. .

C29 C $0.043(2) \quad 0.0059(7) \quad 0.7728(10) 0.129(6)$ Uani $11 \mathrm{~d} .$.

H29 H $-0.0780 \cdot 0.01570 .76480 .155$ Uiso 11 calc $R$.

C30 C $0.0822(14) \quad 0.0730(6) \quad 0.8421(6) \quad 0.082(2)$ Uani $11 \mathrm{~d} . .$.

$\mathrm{H} 30 \mathrm{H} \cdot 0.01190 .09560 .88030 .098$ Uiso $11 \mathrm{calc} R$. .

H2 H $0.297(8) 0.442(4) \quad 0.782(3) \quad 0.049(16)$ Uiso $11 \mathrm{~d} D$.

H3 H $\cdot 0.041(8) 0.383(4) 0.862(4) 0.050(17)$ Uiso $11 \mathrm{~d}$ D.

H1 $1 \mathrm{H} 0.067(7) \quad 0.329(3) \quad 0.507(2) 0.030(13)$ Uiso $11 \mathrm{~d} \mathrm{D}$.

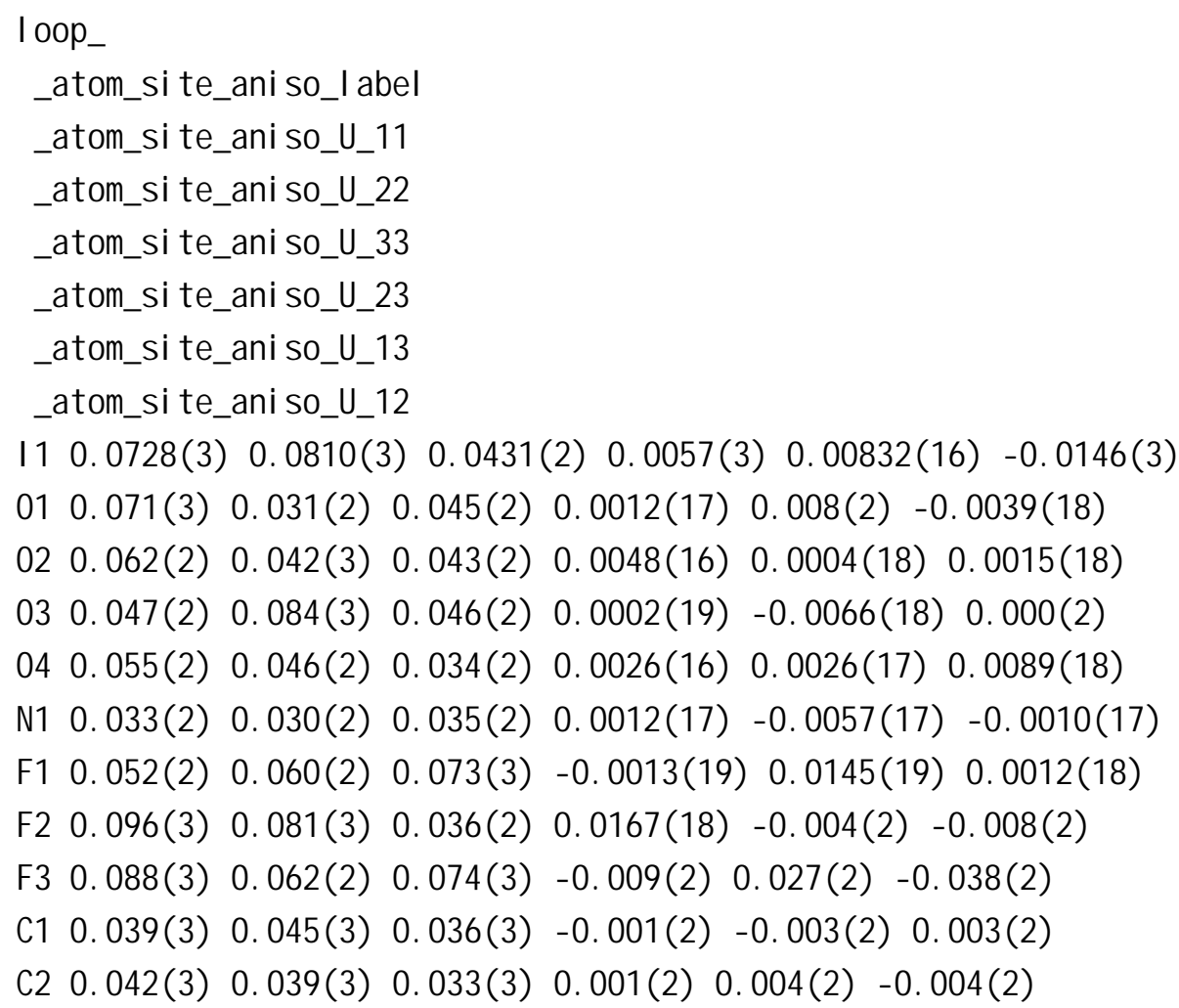




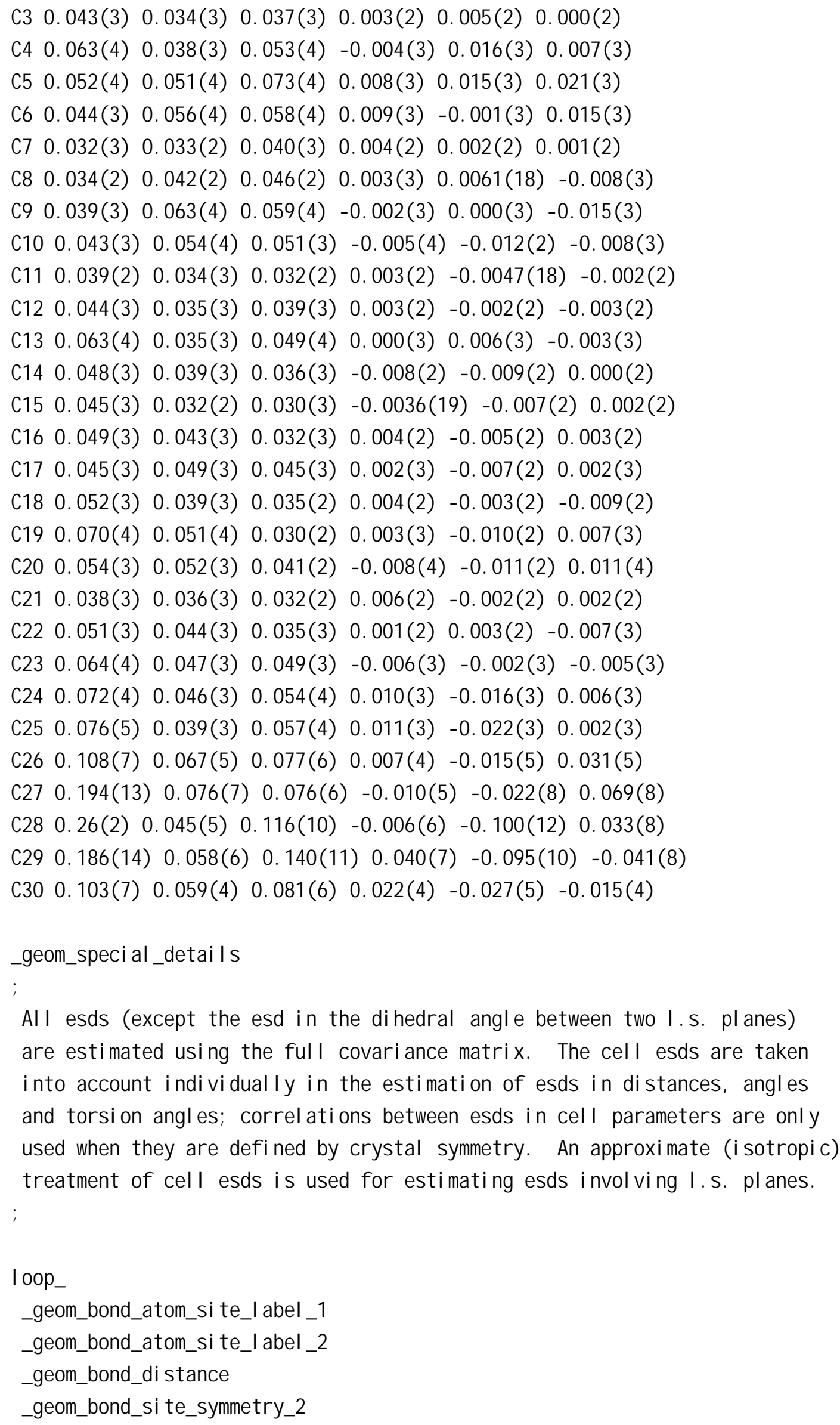


_geom_bond_publ_flag

I1 C18 2.080(5). ?

01 C21 1.205(7). ?

02 C24 1.421(7) . ?

02 C1 1.422(6). ?

$03 \mathrm{C14} 1.193(7)$ ?

$04 \mathrm{C14} 1.342(7)$. ?

$04 \quad 13 \quad 1.445(7)$ ? ?

N1 C21 1.356(7) . ?

N1 C11 1.485(7). ?

N1 C7 1.506(7) . ?

F1 C22 1.320(7) . ?

F2 C22 1.336(7). ?

F3 C22 1.311(7). ?

C1 C2 $1.530(8)$ ?

C1 H1A 0.9700 . ?

C1 H1 B 0.9700 . ?

C2 C3 1.516(8) ?

C2 $C 231.518(8)$ ?

C2 H2 0.96(2). ?

C3 C4 1.534(8) ?

C3 C7 $1.580(7)$. ?

C3 H3 0.96(2) . ?

C4 C5 1.488(10). ?

C4 H4A 0.9700 .?

C4 H4B 0.9700 . ?

C5 C6 $1.510(9)$ ?

C5 H5A 0.9700.?

C5 H5B 0.9700.?

C6 C7 $1.563(8)$ ?

C6 H6A 0.9700 .?

C6 H6B 0.9700 . ?

C7 C8 $1.545(8)$. ?

C8 C9 $1.529(8)$. ?

C8 H8A 0.9700 . ?

C8 H8B 0.9700 . ?

C9 C10 1.517(8). ?

C9 H9A 0.9700 . ?

C9 H9B 0.9700.?

C10 C11 1.529(7). ?

C10 H10A 0.9700.?

C10 H10B 0.9700.?

C11 C12 1.525(8) . ?

C11 H11 0.939(19). ? 


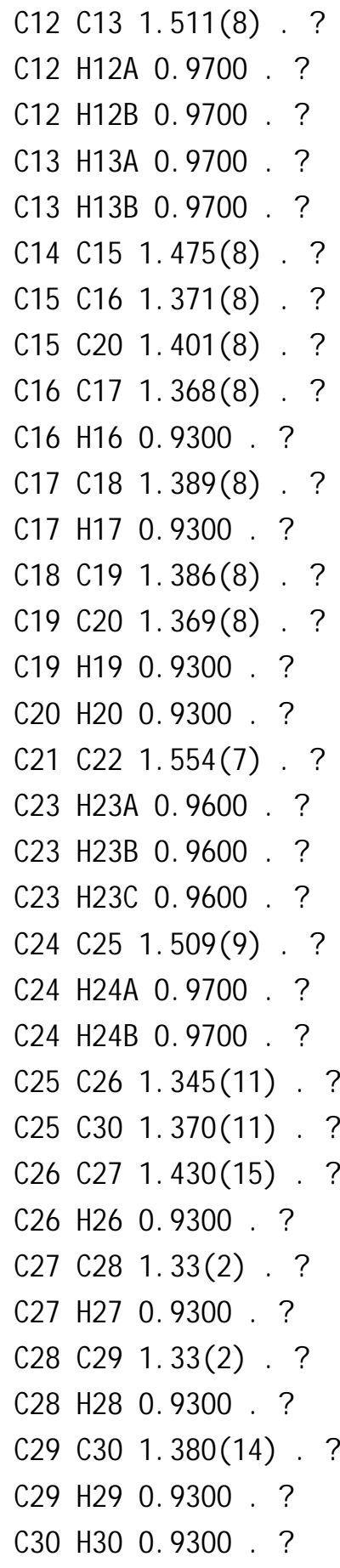

$100 \mathrm{p}$

_geom_angle_atom_site_label_1 _ geom_angle_atom_site_label_2

_ geom_angle_atom_site_label_3

_ geom_angle

_geom_angle_site_symmetry_ 1

_ geom_angle_site_symmetry_3

_ geom_angl e_publ_fl ag

C24 02 C1 113.4(5). . ? 


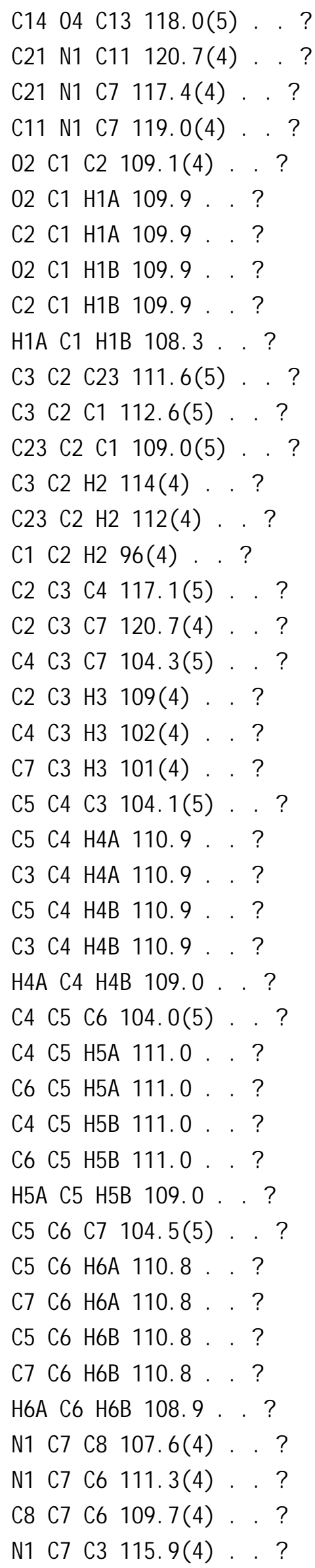




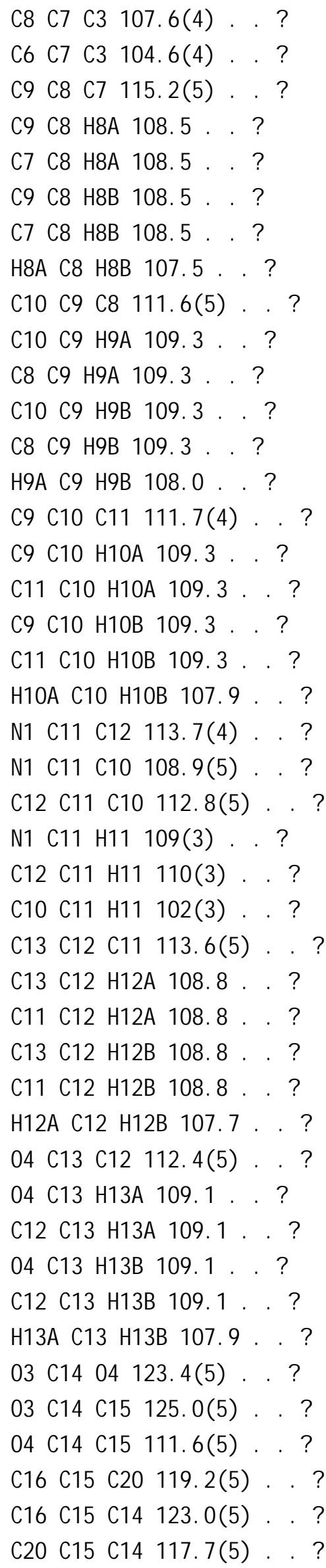




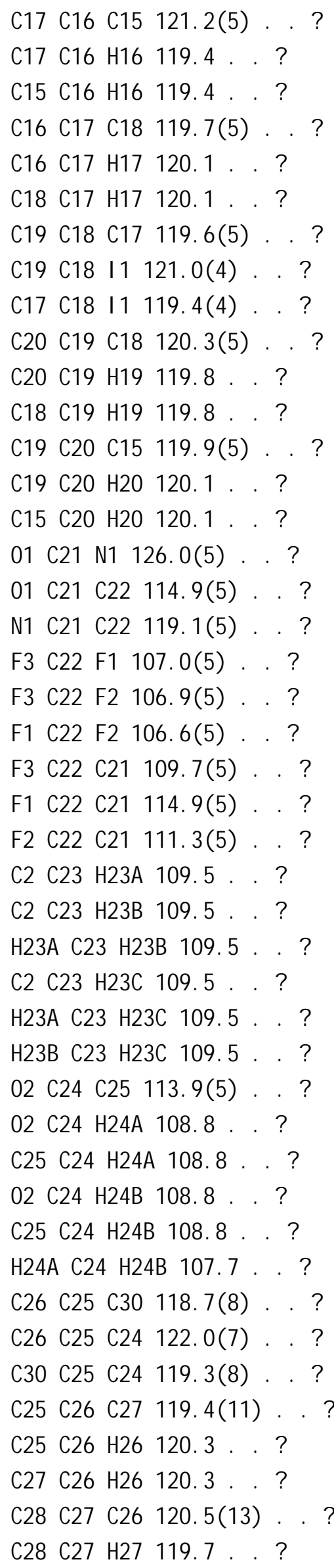




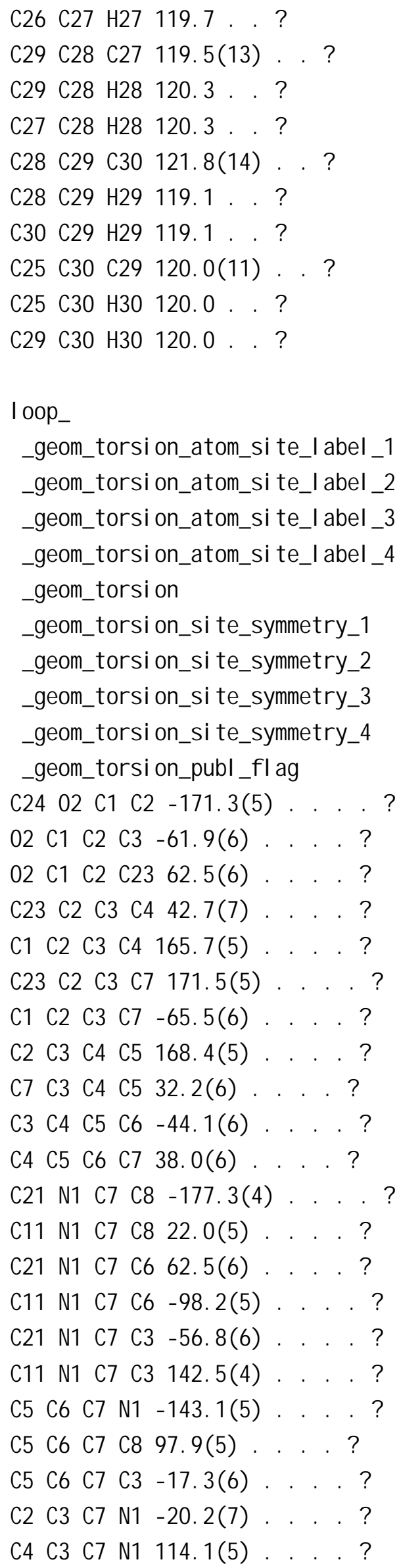




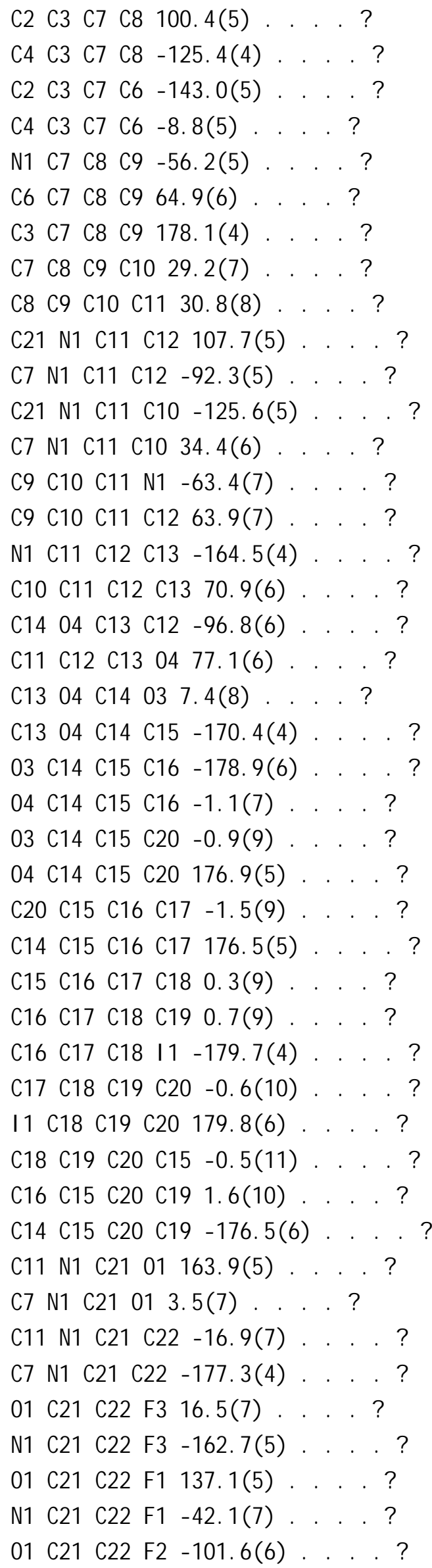


N1 C21 C22 $F 2$ 79.2(6) . . . ?

C1 $02 \quad C 24 C 25 \cdot 69.4(7) \ldots . . ?$

$02 \quad C 24 \quad C 25 \quad C 26 \quad 112.7(7) \ldots . . ?$

$02 \quad 24 \quad C 25 \quad C 30 \cdot 68.3(8) \ldots . . ?$

C30 C25 C26 C27-1.6(11) ... ?

C24 C25 C26 C27 $177.4(7) \ldots \ldots$

C25 C26 C27 C28 3.2(14) . . . ?

C26 C27 C28 C29 $\cdot 2.9(17) \ldots . . ?$

C27 $C 28 \quad C 29 \quad C 301.1(17) \ldots . . \quad$ ?

C26 C25 C30 C29 $\cdot 0.1(11) \ldots . . ?$

C24 C25 C30 C29 $-179.1(7) \ldots . . ?$

C28 $\quad$ C29 $C 30 \quad C 25 \quad 0.4(14) \ldots \ldots$

_diffrn measured_fraction_theta_max $\quad 0.935$

_diffrn_reflns_theta_full 28.31

_diffrn_measured_fraction theta_full 0.935

_refine_diff_density max $\quad 1.295$

_refine_diff_density_mi $n \quad-0.595$

_refine_diff_density_rms 0.114 